\title{
Age-dependent differences in response to partial-thickness cartilage defects in a rat model as a measure to evaluate the efficacy of interventions for cartilage repair
}

\author{
Yorikazu Akatsu ${ }^{1}$. Takahiro Enomoto ${ }^{1}$ - Satoshi Yamaguchi ${ }^{1}$ • Masamichi Tahara ${ }^{2}$ - Taisuke Fukawa ${ }^{1}$. Jun Endo ${ }^{1}$. \\ Hiroko Hoshi ${ }^{1}$ - Yohei Yamamoto ${ }^{1}$ - Toshihide Sasaki ${ }^{1}$ • Kazuhisa Takahashi ${ }^{1}$ - Ryuichiro Akagi ${ }^{1}$ - Takahisa Sasho ${ }^{1,3}$
}

Received: 21 September 2017 / Accepted: 17 August 2018 / Published online: 26 September 2018

(C) The Author(s) 2018

\begin{abstract}
The objectives of this study are (1) to examine age-dependent longitudinal differences in histological responses after creation of partial-thickness articular cartilage defects (PTCDs) in rats and to use this model (2) to objectively evaluate the effectiveness of interventions for cartilage repair. Linear PTCDs were created at a depth of $100 \mu \mathrm{m}$ in the weight-bearing region of the medial femoral condyle in rats of different ages ( 3 weeks, 6 weeks, 10 weeks and 14 weeks). One day, one week, two weeks, four weeks and twelve weeks after PTCD generation, spontaneous healing was evaluated histologically and immunohistochemically. Effects of interventions comprising mesenchymal stem cells (MSCs) or platelet-rich plasma (PRP) or both on 14-week-old PTCD rats were evaluated and compared with natural courses in rats of other ages. Younger rats exhibited better cartilage repair. Cartilage in 3-week-old and 6-week-old rats exhibited nearly normal restoration after 4-12 weeks. Cartilage in 14-week-old rats deteriorated over time and early signs of cartilage degeneration were observed. With injection of MCSs alone or MSCs + PRP, 14-week-old PTCD rats showed almost the same reparative cartilage as 6-week-old rats. With injection of PRP, 14-week-old PTCD rats showed almost the same reparative cartilage as 10-week-old rats. This model will be of great use to objectively compare the effects of interventions for small cartilage lesions and may help to advance the development of disease-modifying osteoarthritis drugs.
\end{abstract}

Keywords Partial-thickness articular cartilage defects $\cdot$ Osteoarthritis $\cdot$ Mesenchymal stem cells $\cdot$ Disease-modifying osteoarthritis drugs $\cdot$ Platelet-rich plasma

\section{Introduction}

Osteoarthritis (OA) of the knee joint detrimentally affects the activities of daily living of the elderly and is costly to manage, resulting in a significant burden on social welfare programs,

Electronic supplementary material The online version of this article (https://doi.org/10.1007/s00441-018-2914-y) contains supplementary material, which is available to authorized users.

Takahisa Sasho

sasho@faculty.chiba-u.jp

1 Department of Orthopedic Surgery, Graduate School of Medicine, Chiba University, 1-8-1 Inohana, Chuo-ku, Chiba 260-8670, Japan

2 Department of Orthopaedic Surgery, Chiba-East-Hospital, Chiba, Japan

3 Center for Preventive Medicine, Musculoskeletal Disease and Pain, Chiba University, Chiba, Japan particularly in countries with aging populations (Buckwalter 2002). Therefore, the establishment of effective prophylactic and treatment options to inhibit the development or progression of OA is long awaited but has not yet been achieved. The major factors involved in OA include the low self-restoration ability of cartilage itself (Buckwalter et al. 2005) and irreversible changes to every tissue in the knee joint once the disease has progressed beyond a certain stage. Thus, it is necessary to explore the feasibility of alternative treatments applicable at the early stage of the disease (Lotz and Caramés 2011), which has proved to be challenging. It is believed that a small cartilaginous defect occurs at the very early stage of OA (Buckwalter 2002; Ding et al. 2005). A cartilage defect is categorized as either a full-thickness cartilage defect (FTCD) or a partial-thickness articular cartilage defect (PTCD). FTCD can be restored by introducing cells from the bone marrow, although this strategy is ineffective for restoration of PTCD 
(Lu et al. 2006; Mankin 1974; Mizuta et al. 2004). Although PTCD is a minor defect compared with FTCD, it does not heal spontaneously and leads to the onset of OA. PTCD on the femoral side induces degeneration of the surrounding cartilage as well as degeneration on the tibial side, followed by progression to OA (Marijnissen et al. 2002; Mastbergen et al. 2006). Recent advancements in magnetic resonance imaging (MRI) techniques allow detection of small cartilage lesions and can be employed to design an early intervention strategy to treat PTCD while it is confined to a limited area.

An earlier study reported that PTCD is restorable during the embryonic stage in experimental animals and in very immature individuals (Namba et al. 1998). We previously reported that PTCD resolved naturally in 3-week-old Sprague Dawley (SD) rats but was incurable in 16-week-old rats (Tsuruoka et al. 2011). Therefore, elucidation of the mechanism causing this difference may directly lead to the development of new therapies for PTCD. However, previous studies failed to define the age at which PTCD is restorable in SD rats.

In the present study, we investigate the natural restoration process of PTCDs in rats of various ages and grade the restoration status by histological analysis. Then, we focus on utilizing this process as an evaluation scale for intervention. In other words, interventions were applied to older rats, which lack restoration ability, to assess the interventional effect by comparison with natural courses observed in younger rats. We opted to use mesenchymal stem cells (MSCs) (Suzuki et al. 2012) and platelet-rich plasma (PRP) (Liu et al. 2014) as the treatment interventions because both have been reported as effective for FTCD and OA, although, to the best of our knowledge, no other study has investigated these interventions for PTCD.

We hypothesize that the younger rats will show better natural restoration of PTCD and age-dependent differences in restoration status can serve as a measure to evaluate the effectiveness of interventions.

\section{Material and methods}

\section{Animals and creation of PTCDs}

Using bilateral knees, 30 knees of 15 male SD rats were assigned to each of four age groups: 3 weeks, 6 weeks, 10 weeks and 14 weeks. Thus, a total of 120 knees were examined. Rats were anesthetized by intraperitoneal injection of ketamine $(0.1 \mathrm{mg} / \mathrm{g})$ and xylazine $(0.01 \mathrm{mg} / \mathrm{g})$. The parapatellar medial approach was used to open the knee joint and a linear PTCD was created in the weight-bearing region of the medial femoral condyle (MFC) in both knees in the direction of joint motion (Yoshioka et al. 1998) by using a specially designed 100- $\mu \mathrm{m}$ scalpel modified from an ophthalmic knife. Subsequently, after performing hemostasis and flushing with saline, the joint capsule and skin were closed with 6-0 nylon sutures. Neither postoperative fixation nor limitation of activity was applied. At 1 day and at 1 week, 2 weeks, 4 weeks and 12 weeks postoperatively, $150 \mu \mathrm{g} / \mathrm{g}$ sodium pentobarbital (Nembutal) was injected intraperitoneally to euthanize the rats and six knees were collected from each group at each time point. In addition, uninjured cartilage of the MFC was examined histologically at day 0 and week 12 in each age group as sham controls. For this purpose, tissue from both knees of three rats (six knees) of each age was harvested.

\section{Effect of MSCs and PRP on PTCD}

According to the results of the first part of the study as well as the results shown by Zhang et al. (2016a, b), 14-week-old male rats, which showed difficulties in natural restoration, were used for intervention studies. PRP was prepared as follows. Rats were anesthetized by intraperitoneal injection of ketamine $(0.1 \mathrm{mg} / \mathrm{g})$ and xylazine $(0.01 \mathrm{mg} / \mathrm{g})$. Then, cardiac puncture was performed to collect blood, which was then injected into a commercial platelet separation kit and centrifuged at $1500 \times g$ for $10 \mathrm{~min}$ (Vetrano et al. 2013). The upper layer was discarded and $1 \mathrm{~mL}$ PRP was prepared from two rats. Platelet concentrations were measured before injection to confirm that the concentration of PRP was increased by 6.4fold from $92 \times 10^{4}$ to $590 \times 10^{4} / \mu \mathrm{L}$.

MSCs were prepared as described elsewhere (Futami et al. 2012). Briefly, synoviums of infra-patellar fat pads were obtained from four 14-week-old rats, washed thoroughly and then digested for $1 \mathrm{~h}$ at $37{ }^{\circ} \mathrm{C}$ with pronase and $3 \mathrm{~h}$ with collagenase. After filtration using a $40-\mu \mathrm{m}$ filter, the sample was cultured in Dulbecco's modified Eagle's medium (Nutrient Mixture F12) supplemented with 20\% fetal bovine serum, $200 \mathrm{mM}$ L-glutamine solution, $50 \mathrm{mg} / \mathrm{mL}$ gentamicin reagent solution and $50 \mu \mathrm{g} / \mathrm{mL}$ ascorbic acid at $37^{\circ} \mathrm{C}$. We changed the culture medium once in 2 days or 3 days, prepared subcultures on day 7 and obtained cells 14 days later (passage 2). To confirm the characteristics of the derived cells, flow cytometry analysis was performed using phycoerythrin (PE) anti-rat CD11b/c, Alexa Fluor ${ }^{\circledR} 647$ anti-rat CD45 and Brilliant Violet 421 $1^{\mathrm{TM}}$ anti-rat CD90/mouse CD90.1 (Thy-1.1) with a Canto II instrument, followed by data analysis using FlowJo, version 9.7.5 (Horie et al. 2009; Yoshimura et al. 2007).

Next, PTCDs were created on the MFCs of 14-week-old SD rats ( $n=12,24$ knees) in the same way as described above. In regard to cell counts, the amount of infusion was determined as mentioned elsewhere (Nishimori et al. 2006). Briefly, the rats were divided into four treatment groups ( $n=3$ each, six knees/group) and $50 \mu \mathrm{L}$ of each of the following solutions was prepared: (a) phosphate-buffered saline (PBS) only, (b) PRP only, (c) $1 \times 10^{6}$ MSCs + PBS and (d) $1 \times 10^{6}$ MSCs + PRP. Immediately after PTCDs, two drops of 
solution containing one of the four interventions were applied to the injured areas and left for $10 \mathrm{~min}$ (Koga et al. 2008). Both knees from a rat received the same intervention. Then, the joint capsule was sutured with 6-0 nylon and the remaining solution was injected into the trans-patella tendon using a 30gauge needle. There was no instance of resistance during injection or leakage from around the wound area or needle in any knee. Four weeks from the interventions rats were euthanized and knees were collected for histological analysis.

\section{Histological and immunohistochemical analyses}

The obtained specimens were soaked in $4 \%$ paraformaldehyde at room temperature for 3 days and, subsequently, in $20 \%$ ethylenediaminetetraacetic acid for 21 days and then embedded and fixed in paraffin, cut into 5- $\mu$ m-thick sections perpendicular to the linear PTCD and stained with Safranin O. The specimens were evaluated using the modified histological system reported by Mukoyama et al. (2015) for assessment of the injured site as well as surrounding area (Table 1). In addition, the deteriorated area surrounding PTCD was assessed by measuring the area of decreased Safranin O staining with ImageJ (National Institutes of Health, USA). The number of cells in $10,000 \mu^{2}$ around PTCDs as well as that of healthy cartilage of the same age were counted and expressed as a ratio of cell numbers with ImageJ. For both analyses, a single slide corresponding to the center of linear PTCD was chosen. The specimens were also immunostained for detection of type II collagen. Briefly, sections were blocked with normal goat

Table 1 Histological score (modified Mukoyama score)

\begin{tabular}{|c|c|c|}
\hline \multirow[t]{4}{*}{ Filling of the defect } & All & 0 \\
\hline & More than $1 / 2$ & 1 \\
\hline & Less than $1 / 2$ & 2 \\
\hline & No repair tissue & 3 \\
\hline \multirow[t]{4}{*}{ Matrix staining (injured area) } & Normal & 0 \\
\hline & Slightly decreased & 1 \\
\hline & Moderately decreased & 2 \\
\hline & No repair tissue & 3 \\
\hline \multirow[t]{4}{*}{ Matrix staining (surrounding area) } & Normal & 0 \\
\hline & Slightly decreased & 1 \\
\hline & Moderately decreased & 2 \\
\hline & Severely decreased & 3 \\
\hline \multirow[t]{4}{*}{ Surface regularity } & Smooth & 0 \\
\hline & Moderate & 1 \\
\hline & Irregular & 2 \\
\hline & No repair tissue & 3 \\
\hline \multirow[t]{4}{*}{ Cell morphology and density (whole area) } & Normal appearance & 0 \\
\hline & Normal but hypocellular & 1 \\
\hline & Abnormal cells & 2 \\
\hline & Absence of cells & 3 \\
\hline
\end{tabular}

serum and incubated with rabbit polyclonal antibody directed against type II collagen (AB2036; Merck Millipore, Darmstadt, Germany). The sections were stained using a 3,3-diaminobenzidine peroxidase substrate kit and visualized with an avidin-biotin peroxidase system (Vectastain ABC Rabbit IgG kit; Vector Laboratories, Burlingame, CA, USA).

All histologic assessments were performed by two histologists who were blinded to the experimental group assignments of samples with 4 years and 5 years of experience, respectively. Ten sections corresponding to the center of the PTCD from a knee were assessed by two examiners and the worst score for each category in Table 1 (higher score) was given to the knee.

\section{Statistical analysis}

For the intrarater reliability of the histological score, one examiner observed the randomly picked up 30 slides and recorded the scores two times at an interval of 1 week. For the interrater reliability, the other examiner observed the same specimens. The intraclass correlation coefficients for intrarater and interrater reliabilities were 0.96 (95\% CI, 0.95-0.97) and 0.92 (95\% CI, 0.82-0.96), respectively.

Data were assessed for a bias of using bilateral knees and effects of age and time after PTCD by using a mixedmodel ANOVA. Then, the Shapiro-Wilk test for normality was used to confirm the absence of normal distribution between each group at each time point. Data were then evaluated using the Kruskal-Wallis test and a nonparametric test (post hoc Steel-Dwass). The MannWhitney $U$ test was used to compare the histological scores of the treatment groups with those of natural courses of different age groups. A probability $(p)$ value $<0.05$ was considered statistically significant.

\section{Results \\ Age-dependent differences in the natural course after PTCD}

Representative histological sections of sham controls stained with Safranin O and immunohistochemical staining for type II collagen are shown in Fig. 1. Representative histological sections of natural-course post-PTCD rats stained with Safranin $\mathrm{O}$ are shown in Fig. 2. Overall, cell density was greater and cell size was smaller in younger rats. Cells lacking nucleus were observed at 1 day after PTCD in the 3-week-old groups but not clear in the other groups. Matrices started to appear in the injured area as early as 1 week after PTCD in the 3-weekold and 6-week-old groups, although Safranin O stainability of the injured area was poor. Almost no restoration was observed in the injured area at 1 week in the 10-week-old and 14- 
3 week-old

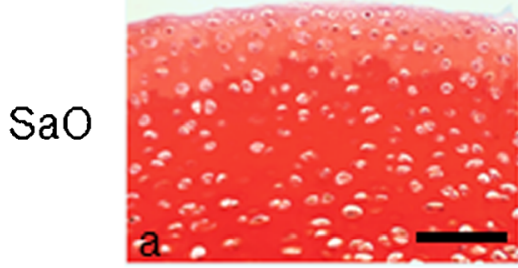

Col-1I

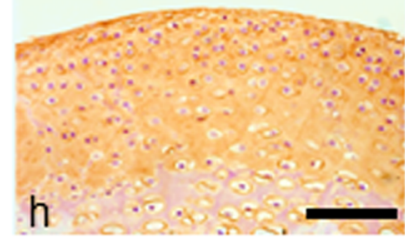

18 week-old
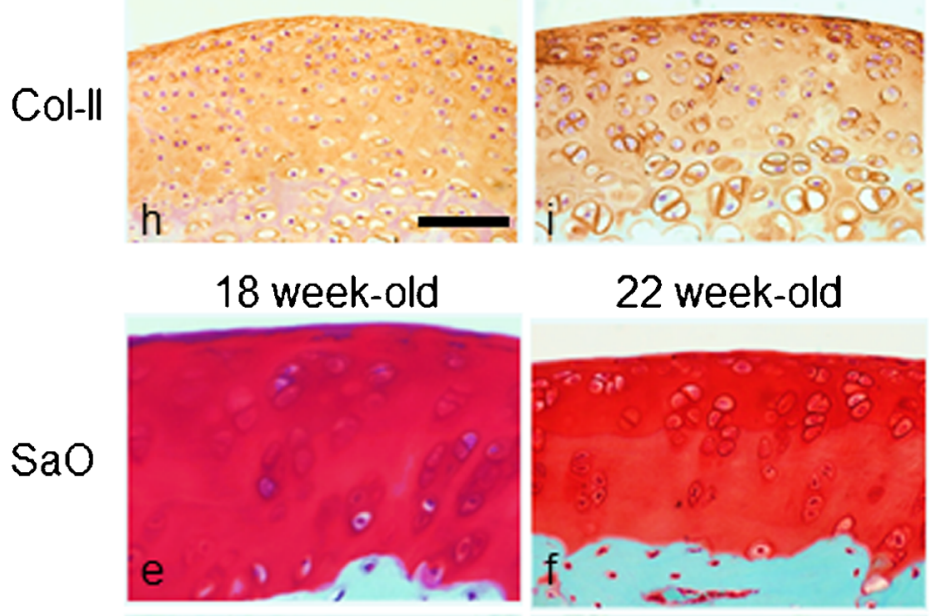

22 week-old

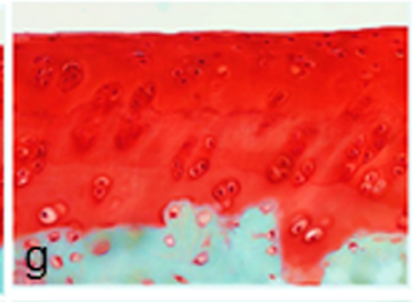

\section{6 week-old}

Col-II
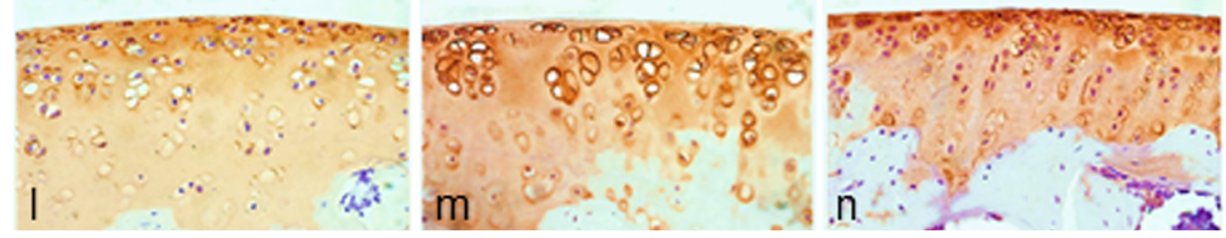

Fig. 1 Histological sections of the sham control cartilage in 3-week-old (a, h), 6-week-old (b, i), 10-week-old (c, j), 14-week-old (d, k), 18-weekold (e, 1), 22-week-old (f, m) and 26-week-old (g, n) rats (a-g Safranin O staining and $\mathrm{h}-\mathrm{n}$ immunohistochemical staining for type II collagen)

week-old groups. In addition, loss of stainability extended into adjacent cartilage of the injured area. Remarkable cartilage restoration and improvement of stainability were observed in the 3-week-old group at 2 weeks after PTCD, although protrusion of the repaired cartilage and decreased stainability of the surface area were observed in the 6-week-old group. In the 10 -week-old and 14-week-old groups, restoration of the injured area was scarce and decreased stainability further extended into the surrounding cartilage in addition to the injured area. At 4 weeks after PTCD, smooth surface was restored with slightly decreased stainability and cluster formation was observed in the 3-week-old group. Likewise, moderate irregularity of the surface of the injured area, cluster formation and slightly decreased stainability were observed in the 6week-old group. On the other hand, the boundary between the injured area and the surrounding area was much clearer and loss of Safranin O stainability in the surrounding area remained in the 10-week-old and 14-week-old groups. At 12 weeks after PTCD, only cluster formation was observed (bar $=100 \mu \mathrm{m}$ ). The youngest rats dealt in the present study were 3 weeks old and the oldest were 26 weeks old because those that are 14 weeks old had been kept for as long as 12 weeks

with good stainability in the 3-week-old group, whereas only cluster formation and mild protrusion of the surface with slightly decreased stainability were observed in the 6-weekold group. In the 10-week-old and 14-week-old groups, the injured area clearly remained and irregularity of the surface and decreased stainability of the surrounding area were observed. Moreover, the decrease in stainability was more pronounced than that at 4 weeks after PTCD. Extension of the unstained area was observed in the 14-week-old group, which was indicative of early degeneration (Mankin and Lippiello 1970).

The histological scores are shown in Fig. 3. The effect of using bilateral knees was not significant $(p=0.145)$. However, the effects of age and time were significant $(p<0.001$ and $p<$ 0.001 , respectively). The scores as early as 1 day after PTCD were not significantly different $(p=0.199)$. Scores were significantly higher at 1 week after PTCD in the 10-week-old and 14week-old groups than in the 3-week-old group ( $p=0.015$ and 0.016 , respectively), and a significant difference was observed 


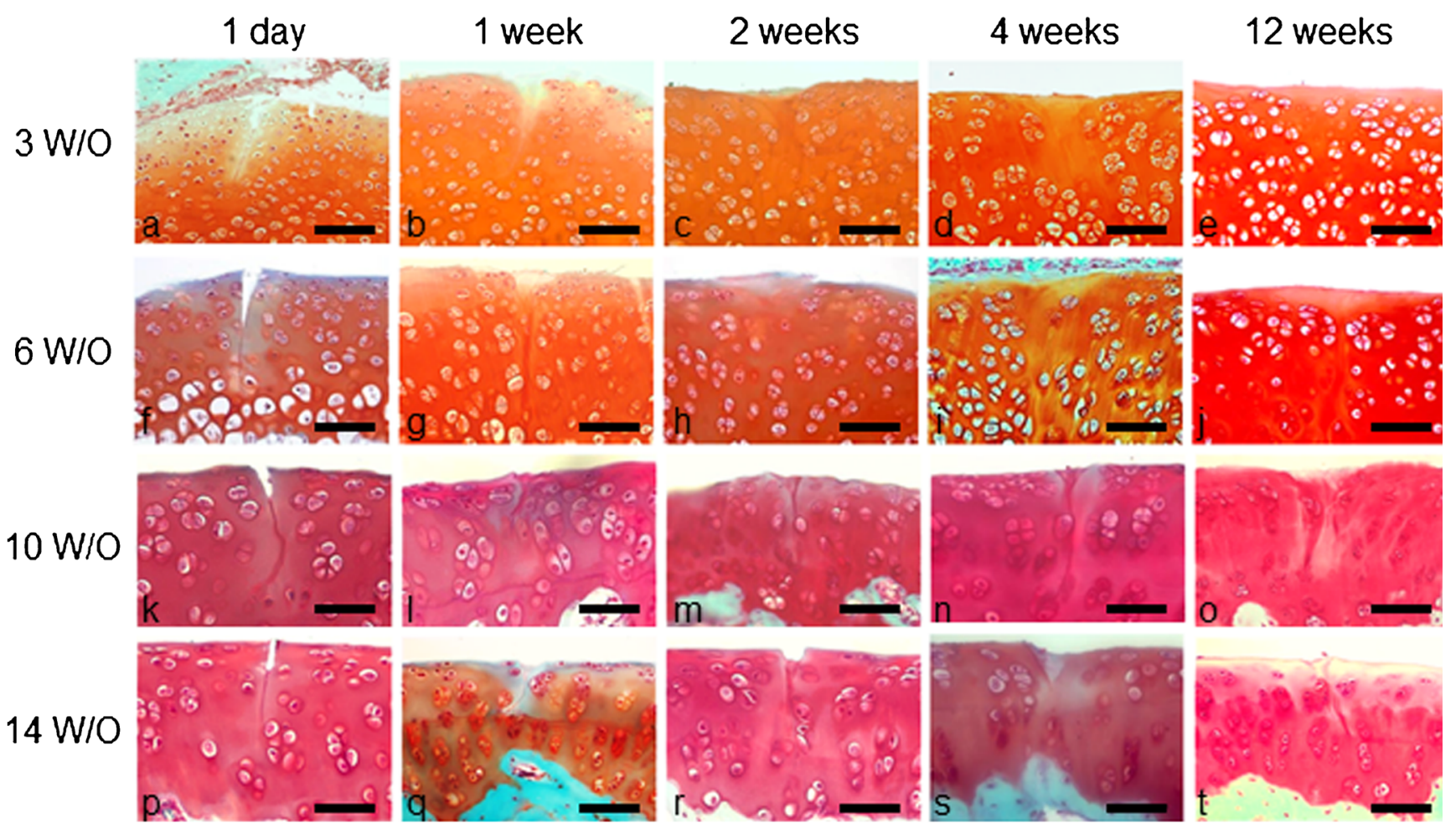

Fig. 2 Histological sections showing the natural course (Safranin O staining) 1 day and 1 week, 2 weeks, 4 weeks and 12 weeks after PTCD in 3-weekold (a-e), 6-week-old (f-j), 10-week-old $(k-0)$ and 14-week-old $(p-t)$ rats $(b a r=100 \mu \mathrm{m})$

between the 6-week-old and 14-week-old groups ( $p=0.018$ ). At 2 weeks after PTCD, the scores in the 10-week-old and 14week-old groups were significantly higher than those in the 3week-old and 6-week-old groups $(p=0.017,0.018,0.017$ and 0.018 , respectively). At 4 weeks after PTCD, scores were significantly lower in the 3-week-old group than in the 6-week-old, 10 -week-old and 14-week-old groups ( $p=0.029,0.014$ and 0.014 , respectively) and in the 6-week-old group than in the 10 -week-old and 14-week-old (W/O) groups ( $p=0.039$ and 0.014 , respectively). At 12 weeks after PTCD, the differences among groups were similar to those at 4 weeks $(p=0.034$, $0.011,0.010,0.029$ and 0.018 , respectively). And, imaging analysis revealed the deteriorated tissue area around PTCD increased as time elapsed in 10-W/O and 14-W/O groups but those from the 3-W/O and 6-W/O groups decreased from 1 week through 12 weeks (Supplement 1). The number of cells had decreased in the 3-W/O group from 1 day to 2 weeks but increased at 4 weeks and returned to normal at 12 weeks. In the 6$\mathrm{W} / \mathrm{O}$ group, a similar tendency was observed in an inconspicuous manner. In the 10-W/O and 14-W/O groups, the number of cells did not change much from 1 day to 4 weeks and an increase was observed at 12 weeks (Supplement 2). Representative images of immunohistochemical staining for type II collagen are shown in Fig. 4. Type II collagen-positive matrices extended to the same extent as the Safranin O-stained areas. In the 3-weekold group, a lack of type II collagen was observed at the injured site at 1 day after PTCD. However, these sites recovered from week 1 through week 4 , when only slight evidence of the injured site remained as a hypocellular area. At 12 weeks, a type II collagen-positive matrix was homogeneously observed and the injured site was undetectable. In the 6-week-old group, loss of type II collagen was observed in the peri-injured area at day 1, which became gradually obscure at week 1 and week 2 . Type II collagen recovered at 4 weeks in the injured sites but the cartilage surface was lacking. In the 10-week-old group, there was no decrease in type II collagen in the peri-injured area at 1 day and minimum loss was observed at 1 week after PTCD, which remained until week 4 and then appeared aggravated at 12 weeks. At the same time, the pericellular matrix started to become strongly positive, which is indicative of cluster formation (Lee et al. 2005; Lotz et al. 2010). In the 14-week-old group, minimal loss of type II collagen was observed in the peri-injured area at 1 week, which aggravated over time and was observed broadly at week 12 . The pericellular matrix was strongly positive from 4 to 12 weeks.

\section{The effect of MSCs and PRP on PTCD as evaluated with a natural-course scale}

From the results of the earlier part of this study, we decided to use 14-week-old SD rats, which showed no repair response, as the recipients of intervention. As shown in Fig. 5, fluorescence-activated cell sorting (FACS) analysis confirmed that the cultured cells were CD11b-negative, CD45-negative and CD90-positive, similar to MSCs. Subsequently, we created PTCDs and performed intra-articular injections in each 


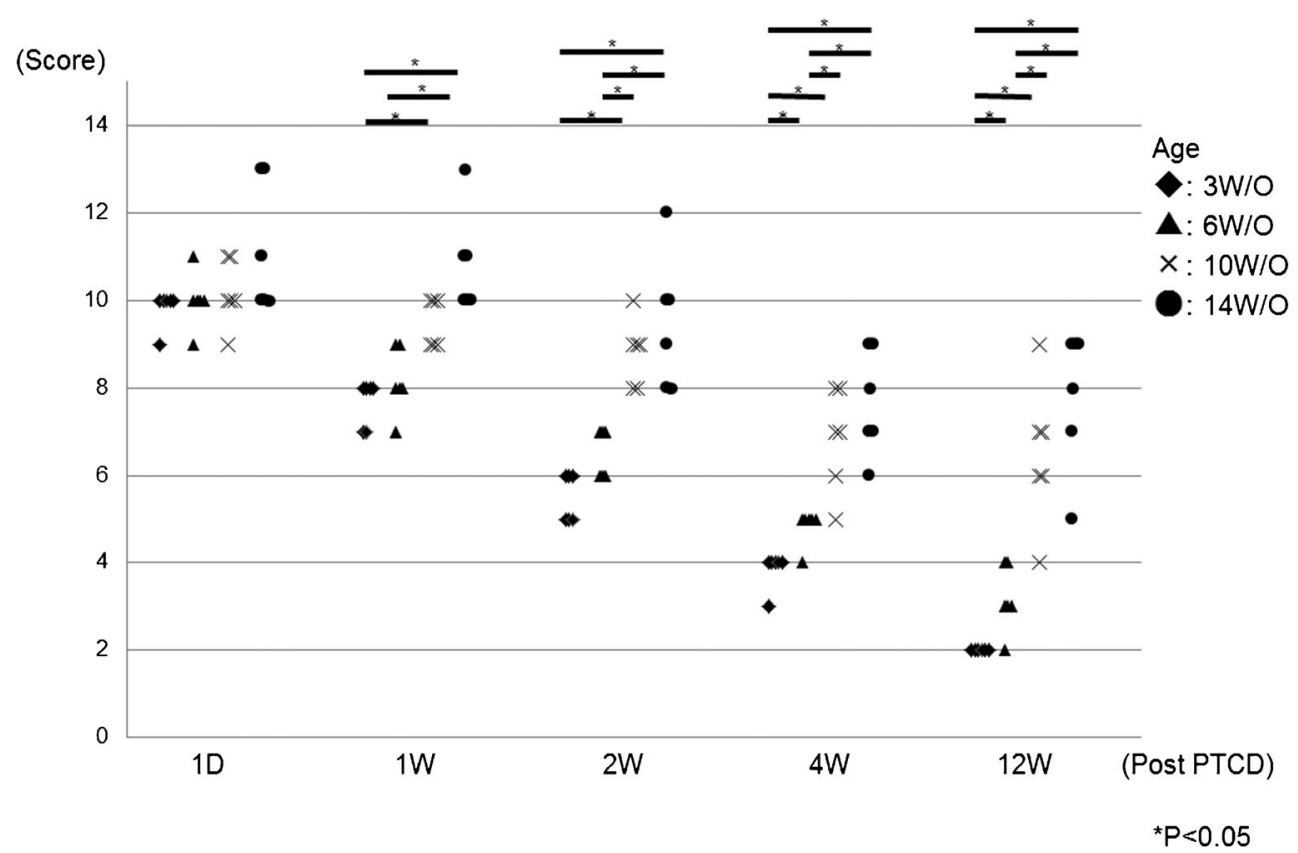

Fig. 3 Histological scores of the natural course after PTCD. Values presented are mean $\pm 95 \%$ confidence interval of the scores $(\%)$ and $(p)$ value. In all groups, no significant differences are observed at 1 day after PTCD $(9.83 \pm 0.41,10.0 \pm 0.63,10.17 \pm 0.75$, and $11.17 \pm 1.47$ in $3-$ week-old, 6-week-old, 10-week-old and 14-week-old rats, respectively; $p=0.199)$. At 1 week after PTCD: 3 -week-old $(7.67 \pm 0.52)$ vs. 6-weekold $(8.17 \pm 0.75)(p=0.586), 10$-week-old $(9.50 \pm 0.55)(p=0.015)$ and 14-week-old $(10.83 \pm 1.17)(p=0.016)$; 6-week-old vs. 10-week-old $(p=$ $0.056)$ and 14-week-old $(p=0.018)$; and 10-week-old vs. 14-week-old $(p=0.091)$ rats. At 2 weeks after PTCD: 3 -week-old (5.50 \pm 0.55$)$ vs. 6 week-old (6.50 \pm 0.55$)(p=0.088), 10$-week-old $(8.83 \pm 0.75)(p=0.017)$

intervention group. There were no infections or complications such as tumor formation. Representative images of Safranin O staining and immunostaining of type II collagen are shown in Fig. 6. In the PBS group, the cartilage surface was irregular and Safranin $\mathrm{O}$ stainability was poor, and tissues similar to those of the natural course in the 14-week-old group at week 4 after PTCD were observed. In the PRP group, the irregularity of the surface was moderate and Safranin O stainability was strong compared with the PBS group and tissues similar to those of the natural course in the 10-week-old group at week 4 after PTCD were observed. In the MSC + PBS group and MSC + PRP group, cartilage restoration was favorable and loss of Safranin O stainability was observed only on the surface and similar tissues to those of the natural course in the 6week-old group at week 4 after PTCD were observed. Lastly, the reparative response was not different between the MSC + PBS group and MSC + PRP group. The histological scores are shown in Fig. 7. The histological scores were significantly higher in the PBS group and PRP group than in the MSC + PBS group and MSC + PRP group $(p=0.020,0.015,0.037$, and 0.015 , respectively) and the findings with the scores matched those described above (PBS group vs. natural 14week-old group: $p=0.773$; PRP group vs. natural 10-week- and 14-week-old $(9.50 \pm 1.52)(p=0.018)$; 6-week-old vs. 10-week-old $(p=0.017)$ and 14-week-old $(p=0.018)$; and 10-week-old vs. 14-weekold $(p=0.875)$ rats. At 4 weeks after PTCD: 3 -week-old $(3.83 \pm 0.41)$ vs. 6-week-old (4.83 \pm 0.41$)(p=0.029), 10$-week-old $(6.83 \pm 1.17)(p=$ $0.014)$ and 14 -week-old $(7.67 \pm 1.21)(p=0.014)$; 6-week-old vs. 10 week-old $(p=0.039)$ and 14-week-old $(p=0.014)$; and 10-week-old vs. 14-week-old $(p=0.707)$ rats. At 12 weeks after PTCD: 3 -week-old $(2.00$ $\pm 0.00)$ vs. 6 -week-old $(3.17 \pm 0.75)(p=0.034), 10$-week-old $(6.50 \pm$ 1.64) $(p=0.011)$ and 14-week-old $(7.83 \pm 1.60)(p=0.010)$; 6-week-old vs. 10 -week-old $(p=0.029)$ and 14 week-old $(p=0.018)$; and 10-weekold vs. 14-week-old $(p=0.500)$ rats

old group: $p=0.572 ;$ MSC + PBS and MSC + PRP groups vs. natural 6-week-old group: $p=0.773$ and 0.586 , respectively).

\section{Discussion}

This is the first study to show differences in the natural restoration ability of PTCDs among different ages of rats and to create a visual scale to evaluate the effectiveness of interventions against incurable PTCDs. Generally, with younger age, better healing of cartilage defects is expected (Wei et al. 1997). Regarding PTCDs, complete healing in fetuses was reported in two papers; however, in postnatal animals, our previous two studies are the only reports of good healing potency against PTCDs in 3-week-old rats (Mukoyama et al. 2015; Tsuruoka et al. 2011) and several other papers have described that PTCD led to the development of OA (Hanie et al. 1992; Mankin 1982). In the present study, an age of less than 6 weeks appeared to be an important factor for healing in rats. Sexual maturation of SD rats may explain this threshold. Long and Evans (1920) reported that the puberty period of SD rats is 50 days after birth on average, while Lewis et al. (2002) considered it as 40-76 days after birth. As there has been no good 


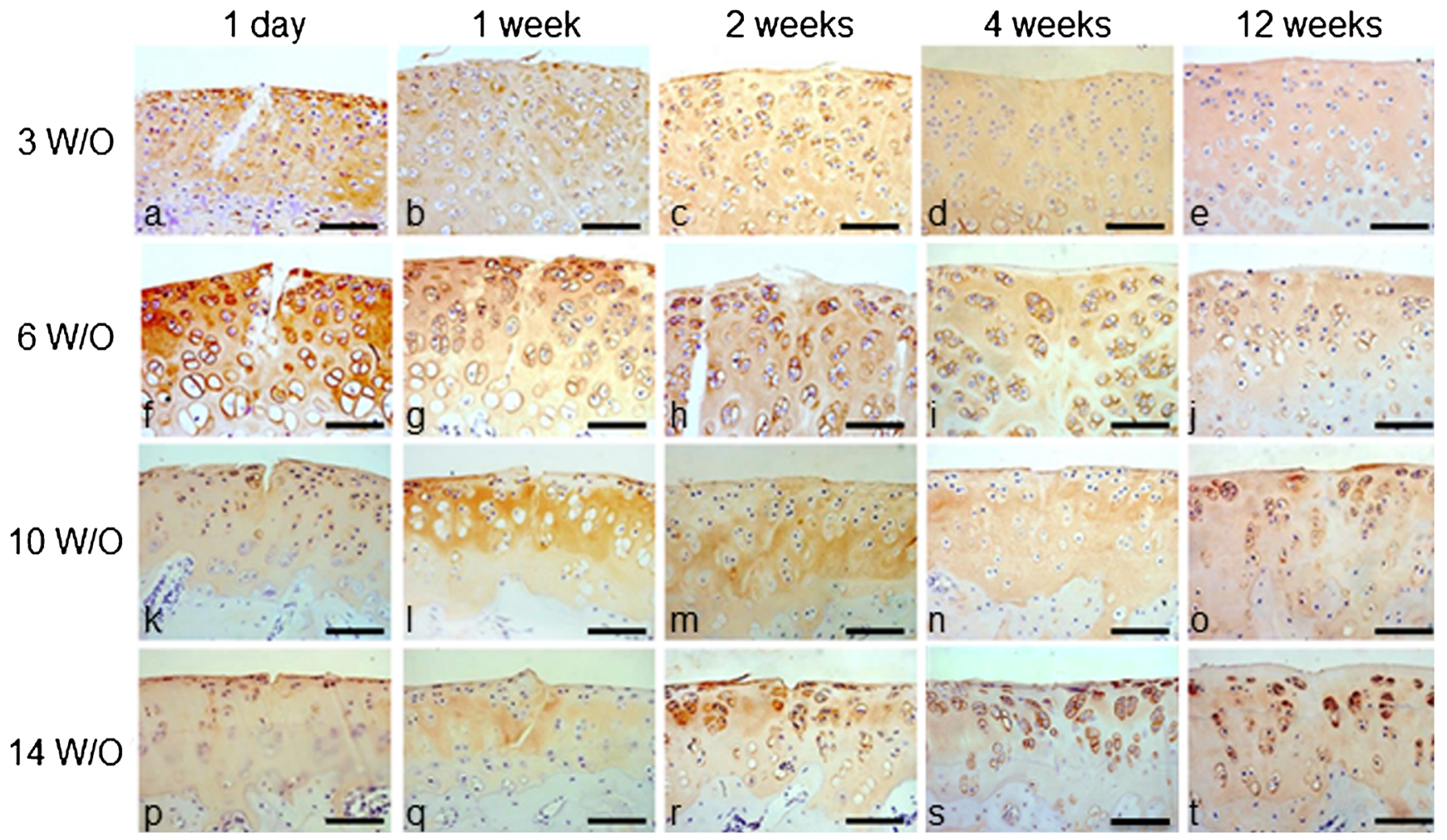

Fig. 4 Immunohistochemical staining for type II collagen shows the natural course after PTCD 1 day and 1 week, 2 weeks, 4 weeks and 12 weeks after PTCD in 3-week-old (a-e), 6-week-old (f-j), 10-week-old (k-o) and 14-week-old $(\mathrm{p}-\mathrm{t})$ groups $(\mathrm{bar}=100 \mu \mathrm{m})$

Fig. 5 FACS analysis of cells used for intra-articular injection. Isotype control (a, $\mathrm{d}, \mathrm{g})$, cells stained with antibody $(\mathrm{b}, \mathrm{e}, \mathrm{h})$ and overlay (c, f, i) of CD11b (a-c), $\mathrm{CD} 45(\mathrm{~d}-\mathrm{f})$ and $\mathrm{CD} 90(\mathrm{~g}-\mathrm{i})$. The cultured cells are CD11b-negative, CD45-negative and CD90positive

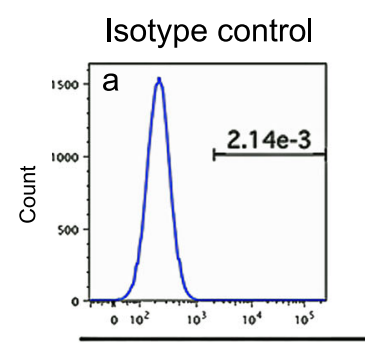

Cells stained with antibody
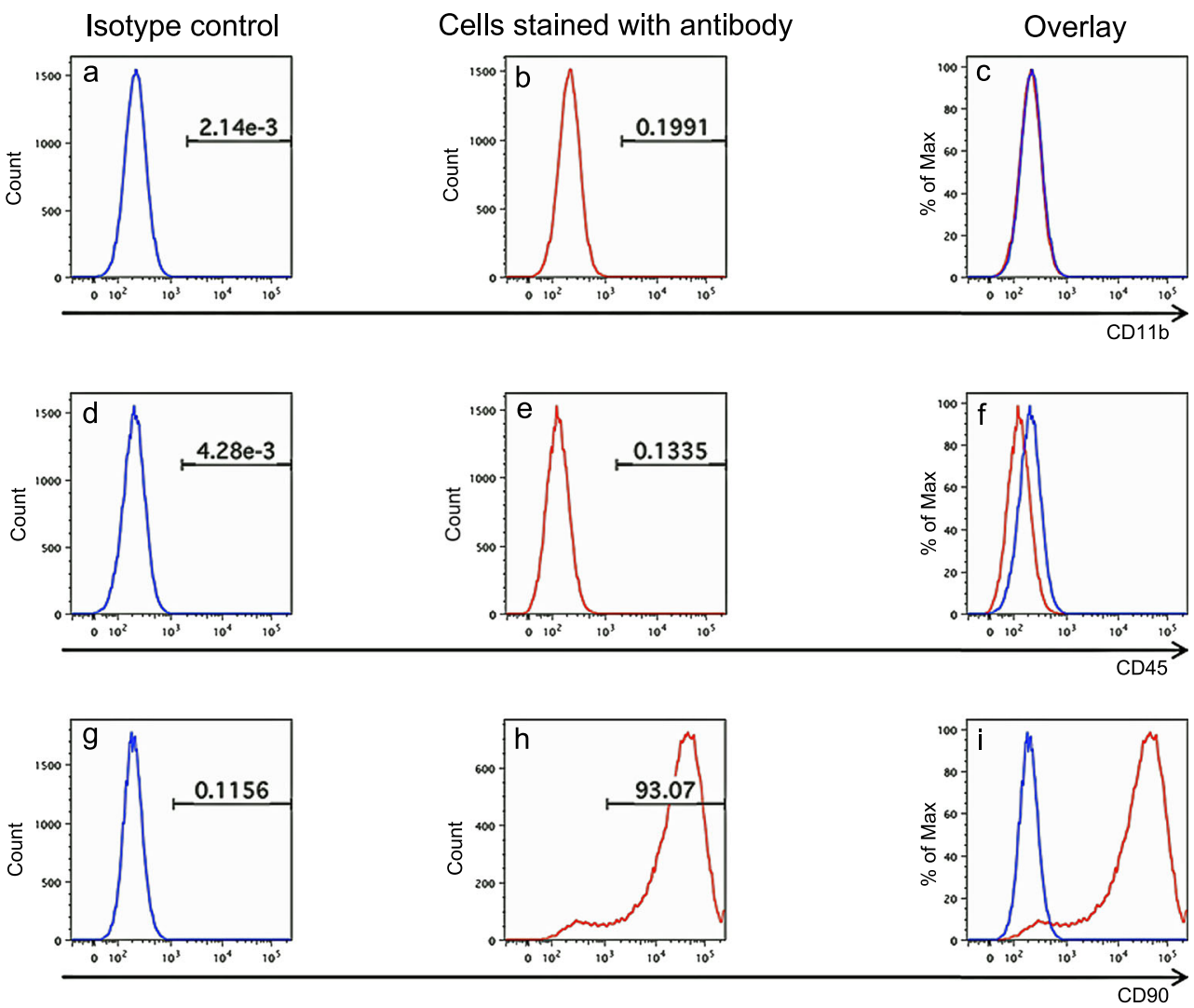


\section{PBS}

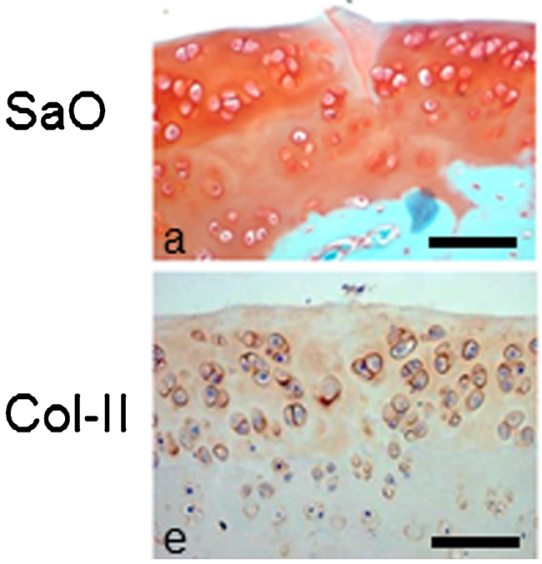

PRP

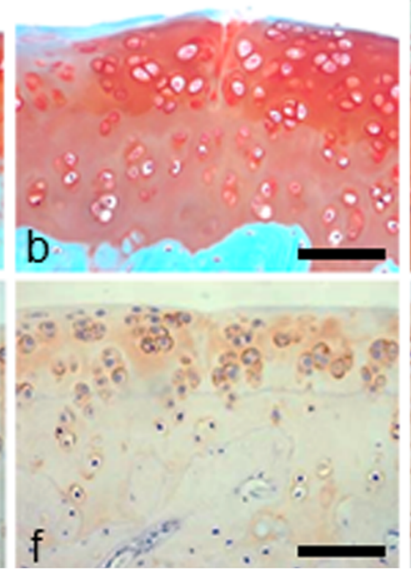

MSCs+PBS

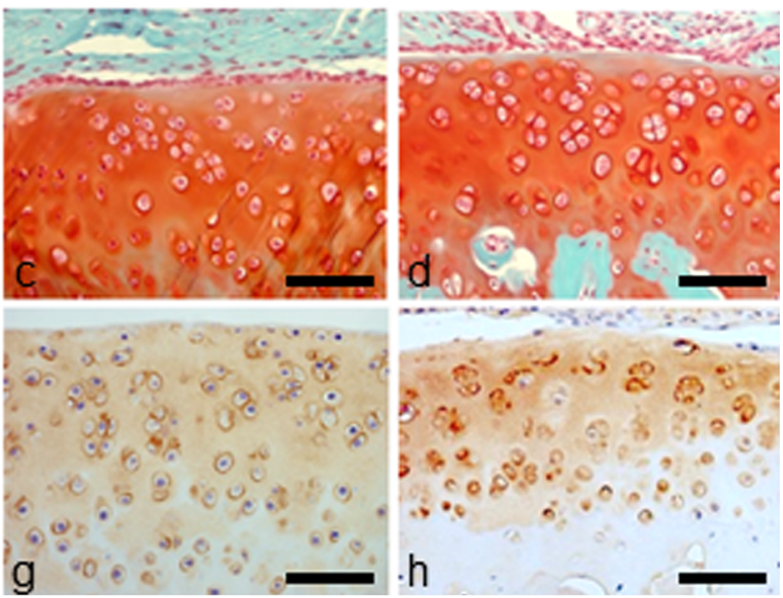

Fig. 6 Histological images of PTCDs in 14-week-old rats following interventions. Safranin O staining (a-d) and immunohistochemical staining (e-h) for type II collagen in the PBS (a, e), PRP (b, f), MSC + PBS (c, g) and MSC + PRP (d, h) groups (bar = $100 \mu \mathrm{m})$

indicator to estimate the skeletal maturity of rats because the epiphyseal plate of rats remains open for life (Chu et al. 2010), it is difficult to elucidate what causes this threshold of musculoskeletal maturity.

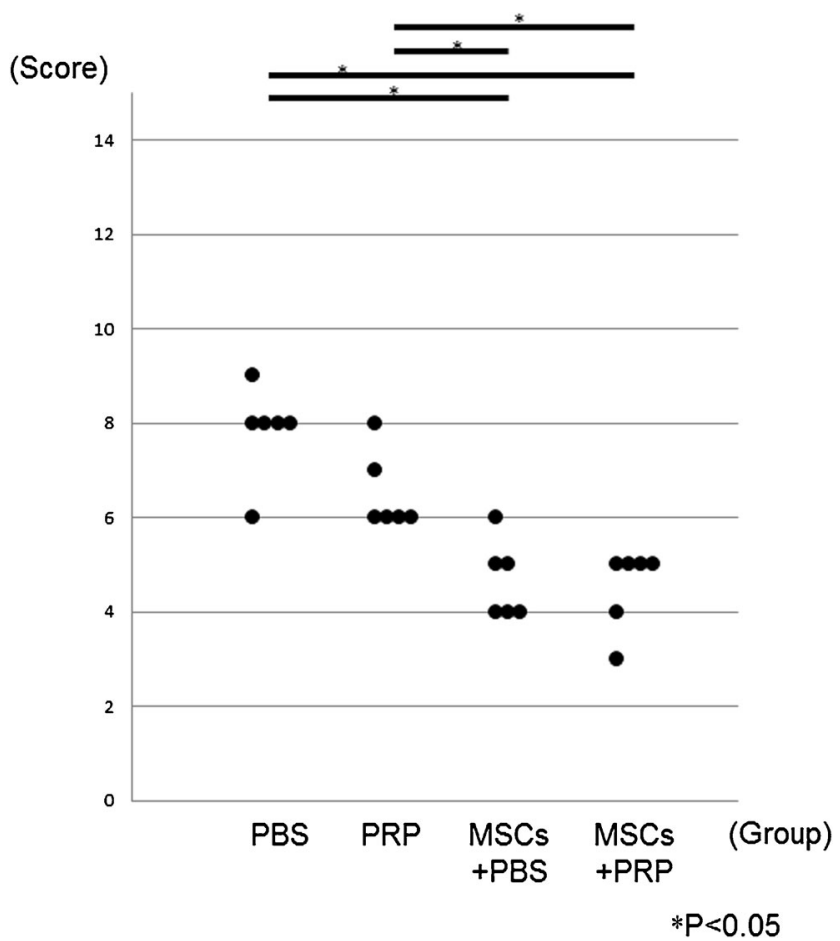

Fig. 7 Histological scores of PTCDs in 14-week-old rats following interventions. Values presented are mean $\pm 95 \%$ confidence interval of the scores $(\%)$ and $(p)$ value between each group. PBS group $(7.83 \pm 0.98)$ vs. PRP group $(6.50 \pm 0.84)(p=0.162), \mathrm{MSC}+\mathrm{PBS}$ group $(4.67 \pm 0.82)$ $(p=0.020)$ and MSC + PRP group (4.50 \pm 0.84$)(p=0.015)$; PRP group vs. MSC + PBS group $(p=0.037)$ and MSC + PRP group $(p=0.015)$; and MSC + PBS group vs. MSC + PRP group $(p=0.999)$
For chondrocytes, different factors due to age have been reported to affect healing potency. Tran-Khanh et al. (2005) reported that the capability to produce extracellular matrix in bovine aged chondrocytes is insufficient compared with younger cells, as evident by proliferation ( \pm twofold less), proteoglycan accumulation per cell $( \pm 20 \%)$ and collagen accumulation per cell $( \pm 55 \%)$. Kumahashi et al. (2004) speculated that abundant ATP receptors and higher ATP activity in fetal chondrocytes may relate to better healing. According to their study, cellular damage and cell death induce outflow of ATP from the cytoplasm, resulting in stimulation of c-fos expression in cells, which controls migration, proliferation and differentiation of cartilage cells at an early stage of cartilage restoration. This appeared to be compatible with the present study in which 3-week-old rats exhibited cells lacking nuclei in the injured sites at an early stage after PTCD. However, young chondrocytes alone may not be sufficient to achieve cartilage repair. Hunziker and Rosenberg (1996), in their study of FTCDs in SD rats, reported that it is important to induce cells from the synovial membrane to restore PTCD. Miyamoto et al. (2007) reported that the synovium plays an important role in cartilage repair. In the present study, we observed synovial adhesion in the injured areas of some younger rats; thus, involvement of synovium may be essential to enhance healing. In addition, synovial-like tissue proliferation and adhesion, which was observed on the surface of PTCDs in the MSC and MSC + PRP groups, might support this idea (Fig. 6). The fact that 3-week-old cartilage PTCDs did not heal spontaneously in an ex vivo system in which an osteochondral plug with PTCD was cultured in media indicated the necessity of the in vivo joint environment including synovium (Mukoyama et al. 2015).

Hunziker and Rosenberg (1996) reported that MSCs play an important role in the restoration of PTCDs and are expected 
to become an effective alternative treatment material because MSCs pose few problems in the donor side with harvesting, easily proliferate in culture and easily differentiate into chondrocytes (Agung et al. 2006; Shirasawa et al. 2006). They speculated migrated MSCs worked as a cell source for repair tissue but recent studies reported an immunomodulatory function of MSCs as another possible mechanism for tissue repair (Nam et al. 2018; Smith et al. 2015). Various tissues have been evaluated as sources of MSCs and synoviumderived stem cells (SDSCs) are considered to have the greatest potential for expansion and chondrogenesis, compared with other MSC sources (De Bari et al. 2001; Sakaguchi et al. 2005). In this study, we used the infra-patellar fat pad as the source of MSCs; these MSCs are easy to collect and have been reported to have performance equal to that of SDSCs and superior to that of muscle-derived cells or bone marrowderived cells (Futami et al. 2012). As successful cartilage regeneration was reported using SDSCs for the treatment of FTCD (Suzuki et al. 2012), we used MSCs in the present study as positive controls for PTCD treatment. The MSC + PRP group was also set up as a possible positive control to obtain better healing. Mifune et al. (2013) reported that the combined use of muscle-derived stem cell (MDSCs) + PRP was significantly favorable for the preservation of cartilage in mono-iodoacetate-induced arthritis of rats compared with the PRP-only group and was more favorable than the MDSC-only group with no statistical significance. As a result, good healing, comparable with that of 6-week-old cartilage, was achieved by administering MDSCs + PRP into PTCD joints. In clinical settings, obtaining synovium for MSCs seems less invasive compared with cartilage repairing techniques such as microfracture, mosaicplasty and autologous chondrocyte implantation but it does require arthroscopy with a small incision. In addition, if we have to adhere to autologous use, the time lag between synovium harvest and MSC preparation may cause loss of the best chance for intervention. A delay of 24 weeks could lead to progression of cartilage degeneration as observed in the 14-week-old rats in our study. Our future goal is to identify molecules or substances that can be applied instantly or with minimum time loss once PTCD is detected. One candidate we considered was PRP, as it contains many growth factors including mainly platelet-derived growth factor, transforming growth factor-beta 1 and insulin-like growth factor (Middleton et al. 2012). Moreover, PRP was reported to contribute to cell proliferation, cell migration, synthesis of ECM-containing collagen, modification of cellular phenotype and arrangement and differentiation of MSCs into cartilage (Schmidt et al. 2006; Weiser et al. 1999). Liu et al. (2014) reported that the use of a rabbit FTCD model with intraarticular injection of PRP every 3 weeks achieved significant cartilage restoration compared with hyaluronan injection, although Serra et al. (2013) reported that PRP injection every 2 days after the creation of FTCD for a total of seven times was not beneficial. In this study, we injected a single dose of PRP after PTCD and modification was observed to the same level as the natural course exhibited by the 10 -week-old group.

PTCD is a post-traumatic early change thought to be identifiable at a high rate clinically (Arøen et al. 2004; Curl et al. 1997). Zhang et al. (2016a, b) reported that about $5 \%$ of arthroscopically treated patients had concomitant PTCD with less than $10 \mathrm{~mm}$ in length and $2 \mathrm{~mm}$ in depth. Because PTCD may progress to irreversible OA (Hunziker and Rosenberg 1996; Mastbergen et al. 2006), it is important to provide effective treatment while the PTCD remains as a microlesion to prevent OA development. The development of diseasemodifying osteoarthritis drugs (DMOADs) is long awaited (Hunter 2011), although there are currently few effective treatments (Qvist et al. 2008). We believe that a good percentage of early OA patients could be spared by finding effective molecules to cure PTCD and that advancements in cartilage imaging techniques with MRI will aid in achieving this goal. Our animal model and histological scale provide solutions for the lack of useful animal models and effective measurement techniques, which have been cited as factors that impede the development of DMOADs (Hunter 2008). The current study provides the following possible solutions for the abovementioned issues: (i) we established a PTCD model that is easy to create using a modified ophthalmic knife, (ii) we clearly showed differences in the effectiveness among interventions using PRP and MSCs so that the effectiveness of these treatments can be better compared and (iii) we showed that a period of 4 weeks is adequate to evaluate the effectiveness of interventions. Our final aim is to develop safer and more effective DMOADs by utilizing the PTCD models presented in this study.

However, this study had the following limitations: (1) the natural restoration was examined only until 12 weeks, (2) joint capsule incision when creating PTCD might have influenced the healing process, (3) the mechanism of cartilage repair and the role of MSCs were not addressed in the present study, (4) the functional role of MSCs was not addressed in the present study, (5) adequate timing and appropriate amounts of MSCs and PRP for injection were not pursued in detail and (6) only four age groups were assessed.

\section{Conclusion}

In this study, we histologically monitored the natural course of PTCD in SD rats of different ages. Then, utilizing the data, we were able to evaluate the effectiveness of cartilage restoration for interventions against PTCD in 14-week-old SD rats. We believe this model will be of great use in the future of DMOAD development. 
Funding This work was supported by Veritas, Ltd. (Tokyo, Japan) and a Grant-in Aid for Scientific Research (C) of the Japan Society for the Promotion of Science (JPJS), Grant Number 2591675.

\section{Compliance with ethical standards}

Conflict of interest The authors declare that they have no conflict of interest.

Research involving animals The study protocol was approved by the institutional Ethics Committee of Chiba University and all experiments were conducted in the animal experiment facility of Chiba University.

Open Access This article is distributed under the terms of the Creative Commons Attribution 4.0 International License (http:// creativecommons.org/licenses/by/4.0/), which permits unrestricted use, distribution and reproduction in any medium, provided you give appropriate credit to the original author(s) and the source, provide a link to the Creative Commons license and indicate if changes were made.

\section{References}

Agung M, Ochi M, Yanada S, Adachi N, Izuta Y, Yamasaki T, Toda K (2006) Mobilization of bone marrow-derived mesenchymal stem cells into the injured tissues after intraarticular injection and their contribution to tissue regeneration. Knee Surg Sports Traumatol Arthrosc 14:1307-1314

Arøen A, Løken S, Heir S, Alvik E, Ekeland A, Granlund OG, Engebretsen L (2004) Articular cartilage lesions in 993 consecutive knee arthroscopies. Am J Sports Med 32:211-215

Buckwalter JA (2002) Articular cartilage injuries. Clin Orthop Relat Res: $21-37$

Buckwalter JA, Mankin HJ, Grodzinsky AJ (2005) Articular cartilage and osteoarthritis. Instr Course Lect 54:465-480

Chu CR, Szczodry M, Bruno S (2010) Animal models for cartilage regeneration and repair. Tissue Eng Part B Rev 16:105-115

Curl WW, Krome J, Gordon ES, Rushing J, Smith BP, Poehling GG (1997) Cartilage injuries: a review of 31,516 knee arthroscopies. Arthroscopy 13:456-460

De Bari C, Dell'Accio F, Tylzanowski P, Luyten FP (2001) Multipotent mesenchymal stem cells from adult human synovial membrane. Arthritis Rheum 44:1928-1942

Ding C, Cicuttini F, Scott F, Boon C, Jones G (2005) Association of prevalent and incident knee cartilage defects with loss of tibial and patellar cartilage: a longitudinal study. Arthritis Rheum 52:39183927

Futami I, Ishijima M, Kaneko H, Tsuji K, Ichikawa-Tomikawa N, Sadatsuki R, Muneta T, Arikawa-Hirasawa E, Sekiya I, Kaneko K (2012) Isolation and characterization of multipotential mesenchymal cells from the mouse synovium. PLoS One 7:e45517

Hanie EA, Sullins KE, Powers BE, Nelson PR (1992) Healing of fullthickness cartilage compared with full-thickness cartilage and subchondral bone defects in the equine third carpal bone. Equine Vet J 24:382-386

Horie M, Sekiya I, Muneta T, Ichinose S, Matsumoto K, Saito H, Murakami T, Kobayashi E (2009) Intra-articular injected synovial stem cells differentiate into meniscal cells directly and promote meniscal regeneration without mobilization to distant organs in rat massive meniscal defect. Stem Cells 27:878-887

Hunter DJ (2008) Are there promising biologic therapies for osteoarthritis? Curr Rheumatol Rep 10:19-25

Hunter DJ (2011) Pharmacologic therapy for osteoarthritis - the era of disease modification. Nat Rev Rheumatol 7:13-22
Hunziker EB, Rosenberg LC (1996) Repair of partial-thickness defects in articular cartilage: cell recruitment from the synovial membrane. J Bone Joint Surg Am 78:721-733

Koga H, Shimaya M, Muneta T, Nimura A, Morito T, Hayashi M, Suzuki S, Ju YJ, Mochizuki T, Sekiya I (2008) Local adherent technique for transplanting mesenchymal stem cells as a potential treatment of cartilage defect. Arthritis Res Ther 10:R84

Kumahashi N, Ochi M, Kataoka H, Uchio Y, Kakimaru H, Sugawara K, Enomoto K (2004) Involvement of ATP, increase of intracellular calcium and the early expression of c-fos in the repair of rat fetal articular cartilage. Cell Tissue Res 317:117-128

Lee JE, Jeong MH, Ahn HJ, Kim JK, Choi K, Chang CB, Kim HJ, Seong SC, Lee MC (2005) Evaluation of chondrogenesis in collagen/chitosan/glycosaminoglycan scaffolds for cartilage tissue engineering. Tissue Engineering Regenerative Medicine 2:41-49

Lewis EM, Barnett JF, Freshwater L, Hoberman AM, Christian MS (2002) Sexual maturation data for Crl Sprague-Dawley rats: criteria and confounding factors. Drug Chem Toxicol 25:437-458

Liu J, Song W, Yuan T, Xu Z, Jia W, Zhang C (2014) A comparison between platelet-rich plasma (PRP) and hyaluronate acid on the healing of cartilage defects. PLoS One 9:e97293

Long J, Evans A (1920) On the attainment of sexual maturity and the character of the first estrous cycle in the rat. Anat Recrd 18:244-245

Lotz MK, Caramés B (2011) Autophagy and cartilage homeostasis mechanisms in joint health, aging and OA. Nat Rev Rheumatol 7:579587

Lotz MK, Otsuki S, Grogan SP, Sah R, Terkeltaub R, D’Lima D (2010) Cartilage cell clusters. Arthritis Rheum 62:2206-2218

Lu Y, Markel MD, Swain C, Kaplan LD (2006) Development of partial thickness articular cartilage injury in an ovine model. J Orthop Res 24:1974-1982

Mankin HJ, Lippiello L (1970) Biochemical and metabolic abnormalities in articular cartilage from osteo-arthritic human hips. J Bone Joint Surg Am 52:424-434

Mankin HJ (1974) The reaction of articular cartilage to injury and osteoarthritis (first of two parts). N Engl J Med 291:1285-1292

Mankin HJ (1982) The response of articular cartilage to mechanical injury. J Bone Joint Surg Am 64:460-466

Marijnissen AC, van Roermund PM, Verzijl N, Tekoppele JM, Bijlsma JW, Lafeber FP (2002) Steady progression of osteoarthritic features in the canine groove model. Osteoarthr Cartil 10:282-289

Mastbergen SC, Marijnissen AC, Vianen ME, van Roermund PM, Bijlsma JW, Lafeber FP (2006) The canine 'groove' model of osteoarthritis is more than simply the expression of surgically applied damage. Osteoarthr Cartil 14:39-46

Middleton KK, Barro V, Muller B, Terada S, Fu FH (2012) Evaluation of the effects of platelet-rich plasma (PRP) therapy involved in the healing of sports-related soft tissue injuries. Iowa Orthop J 32: $150-163$

Mifune Y, Matsumoto T, Takayama K, Ota S, Li H, Meszaros LB, Usas A, Nagamune K, Gharaibeh B, Fu FH, Huard J (2013) The effect of platelet-rich plasma on the regenerative therapy of muscle derived stem cells for articular cartilage repair. Osteoarthr Cartil 21:175-185

Miyamoto A, Deie M, Yamasaki T, Nakamae A, Shinomiya R, Adachi N, Ochi M (2007) The role of the synovium in repairing cartilage defects. Knee Surg Sports Traumatol Arthrosc 15:1083-1093

Mizuta H, Kudo S, Nakamura E, Otsuka Y, Takagi K, Hiraki Y (2004) Active proliferation of mesenchymal cells prior to the chondrogenic repair response in rabbit full-thickness defects of articular cartilage. Osteoarthr Cartil 12:586-596

Mukoyama S, Sasho T, Akatsu Y, Yamaguchi S, Muramatsu Y, Katsuragi J, Fukawa T, Endo J, Hoshi H, Yamamoto Y, Takahashi K (2015) Spontaneous repair of partial thickness linear cartilage injuries in immature rats. Cell Tissue Res 359:513-520 
Nam Y, Rim YA, Lee J, Ju JH (2018) Current therapeutic strategies for stem cell-based cartilage regeneration. Stem Cells Int Mar 2018(8490489):25

Namba RS, Meuli M, Sullivan KM, Le AX, Adzick NS (1998) Spontaneous repair of superficial defects in articular cartilage in a fetal lamb model. J Bone Joint Surg Am 80:4-10

Nishimori M, Deie M, Kanaya A, Exham H, Adachi N, Ochi M (2006) Repair of chronic osteochondral defects in the rat. A bone marrowstimulating procedure enhanced by cultured allogenic bone marrow mesenchymal stromal cells. J Bone Joint Surg Br 88:1236-1244

Qvist P, Bay-Jensen AC, Christiansen C, Dam EB, Pastoureau P, Karsdal MA (2008) The disease modifying osteoarthritis drug (DMOAD): is it in the horizon? Pharmacol Res 58:1-7

Sakaguchi Y, Sekiya I, Yagishita K, Muneta T (2005) Comparison of human stem cells derived from various mesenchymal tissues: superiority of synovium as a cell source. Arthritis Rheum 52:2521-2529

Schmidt MB, Chen EH, Lynch SE (2006) A review of the effects of insulin-like growth factor and platelet derived growth factor on in vivo cartilage healing and repair. Osteoarthr Cartil 14:403-412

Serra CI, Soler C, Carrillo JM, Carillo JM, Sopena JJ, Redondo JI, Cugat R (2013) Effect of autologous platelet-rich plasma on the repair of full-thickness articular defects in rabbits. Knee Surg Sports Traumatol Arthrosc 21:1730-1736

Shirasawa S, Sekiya I, Sakaguchi Y, Yagishita K, Ichinose S, Muneta T (2006) In vitro chondrogenesis of human synovium-derived mesenchymal stem cells: optimal condition and comparison with bone marrow-derived cells. J Cell Biochem 97:84-97

Smith B, Sigal IR, Grande DA (2015) Immunology and cartilage regeneration. Immunol Rea 63:181-186

Suzuki S, Muneta T, Tsuji K, Ichinose S, Makino H, Umezawa A, Sekiya I (2012) Properties and usefulness of aggregates of synovial mesenchymal stem cells as a source for cartilage regeneration. Arthritis Res Ther 14:R136
Tran-Khanh N, Hoemann CD, McKee MD, Henderson JE, Buschmann MD (2005) Aged bovine chondrocytes display a diminished capacity to produce a collagen-rich, mechanically functional cartilage extracellular matrix. J Orthop Res 23:1354-1362

Tsuruoka H, Sasho T, Yamaguchi S, Ikegawa N, Saito M, Akagi R, Ochiai N, Nakagawa K, Nakajima A, Fallouh L, Takahashi K (2011) Maturation-dependent spontaneous healing of partial thickness cartilage defects in infantile rats. Cell Tissue Res 346:263-271

Vetrano M, Castorina A, Vulpiani MC, Baldini R, Pavan A, Ferretti A (2013) Platelet-rich plasma versus focused shock waves in the treatment of jumper's knee in athletes. Am J Sports Med 41:795-803

Wei X, Gao J, Messner K (1997) Maturation-dependent repair of untreated osteochondral defects in the rabbit knee joint. J Biomed Mater Res 34:63-72

Weiser L, Bhargava M, Attia E, Torzilli PA (1999) Effect of serum and platelet-derived growth factor on chondrocytes grown in collagen gels. Tissue Eng 5:533-544

Yoshimura H, Muneta T, Nimura A, Yokoyama A, Koga H, Sekiya I (2007) Comparison of rat mesenchymal stem cells derived from bone marrow, synovium, periosteum, adipose tissue, and muscle. Cell Tissue Res 327:449-462

Yoshioka M, Kubo T, Coutts RD, Hirasawa Y (1998) Differences in the repair process of longitudinal and transverse injuries of cartilage in the rat knee. Osteoarthr Cartil 6:66-75

Zhang K, Shi J, Li Y, Jiang Y, Tao T, Li W, Gui J (2016a) Chondrogenic cells respond to partial-thickness defects of articular cartilage in adult rats: an in vivo study. J Mol Histol 47:249-258

Zhang X, You T, Jiang X, Zhang H, Zhang W (2016b) Characteristic arthroscopic signs of cartilage injuries indicating concomitant occult medial meniscal peripheral tears of posterior horn. Int Orthop 40: $191-195$ 